\title{
3D QSAR CoMFA Study on Phenylthiazolylhydrazide (PTH) Derivataives as Tau Protein Aggregation Inhibitors
}

\author{
Hye Ri Park, Mi Kyoung Kim, Dong Woon Kim, ${ }^{\dagger}$ IL Han Choo, ${ }^{\ddagger, *}$ and Youhoon Chong* \\ Department of Bioscience and Biotechnology, Bio/Molecular Informatics Center, Konkuk University, \\ Seoul 143-701, Korea. *E-mail: chongy@konkuk.ac.kr \\ ${ }^{\dagger}$ Swine Science Division, National Institute of Animal Science, RDA, Cheonan 330-801, Korea \\ "Department of Neuropsychiatry, Seoul National University Hospital, Seoul 110-744, Korea.*E-mail: npchoo10@snu.ac.kr \\ Received July 28, 2010, Accepted September 29, 2010
}

Key Words: Tau protein, Alzheimer, 3D-QSAR, CoMFA

The pathological hallmarks of Alzheimer's disease (AD) are two types of aggregates in brain: extracellular amyloid plaques consisting of the $\mathrm{A} \beta$ peptide $^{1}$ and intracellular neurofibrillary tangles (NFTs). ${ }^{2-4}$ Abnormal filaments called paired helical filaments (PHFs) are major components of NFTs, and a microtubule-associated protein tau consists of its core protein. Even though the primary cause of these aggregation processes in not well-understood, there is a general consensus that protein aggregates are toxic for neurons and a pathological series of neurotoxic events lead to neurodegeneration and finally AD. Therefore, inhibition of the aggregation process by a small molecule has been intensively studied as a therapeutic intervention for $\mathrm{AD}$. Even though $\mathrm{A} \beta$ inhibitors have been a major focus of research on anti-Alzheimer therapy, ${ }^{5}$ recent report of a phase II clinical trial of the tau aggregation inhibitor MTC (methylthionium chloride) which significantly slowed down the decline of cognitive functions of $\mathrm{AD}$ patients ${ }^{6}$ proposes a valuable therapeutic use of the tau aggregation inhibitor as a potential disease-modifying drug for $\mathrm{AD}$. Interests towards inhibitors of the tau aggregation continues to grow by recent findings of several classes of inhibitors such as rhodanines, ${ }^{7}$ phenylthiazolylhydrazides, ${ }^{8-10} \mathrm{~N}$-phenylamines, ${ }^{11}$ anthraquinones, ${ }^{12}$ benzothiazoles, ${ }^{13}$ phenothiazines, ${ }^{14}$ porphyrins ${ }^{14}$ and polyphenols. ${ }^{14}$ However, the low membrane permeability of most of the aggregation inhibitors is the key issue which needs to be resolved for future development of new generation of aggregation inhibitors. In this regard, as most potent inhibitors including MTC are negatively or positively charged, the neutral phenylthiazolylhydrazides (PTH, Fig. 1) drew our specific attention.

The PTH derivatives show in vitro nanomolar $\mathrm{IC}_{50}$ values for tau aggregation inhibition ${ }^{10}$ but, presumably due to the presence of the hydrazothiazole toxicophore, ${ }^{15}$ the PTH derivatives are relatively cytotoxic. ${ }^{10}$ Thus, in this study, we report a 3DQSAR study on the PTH derivatives in anticipation of getting a model that would account for the quantitative differences in bioactivity seen in this series and provide insights into designing

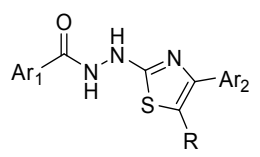

Figure 1. Structure of phenylthiazolylhydrazides (PTH). of novel tau aggregation inhibitors with improved activity.

The in vitro biological activity data reported as $\mathrm{IC}_{50}$ for inhibition of tau aggregation by the PTH derivatives ${ }^{10}$ were used for the current study. Out of total 48 PTH derivatives, 20 compounds lacking inhibitory activity in exact numerical form have been removed from the analysis. During the rigorous cycle of model development and validation, we found four compounds not to fit to either the training set or test set. These outliers probably acting via a different mechanism were also dropped and 27 compounds were left for our study (Table 1).

The data set was randomly separated into a training set (22 compounds) for which 3D-QSAR models were derived and a test set (5 compounds) that would prove the external predictivity of the resulting model. Compound number with * in Table 1 stands for molecules included in the test set.

All calculations were carried out on a linux enterprise operating system using molecular modeling software package SYBYL v 7.2. ${ }^{16}$ The PTH derivatives were sketched, assigned with Gästeiger-Hückel charges, and energy-minimized with Tripos force field. In order to locate the most stable rotamer around the hydrazide $\mathrm{N}-\mathrm{N}$ bond, systematic searches were performed with an interval of $10^{\circ}$. The most crucial step in performing $3 \mathrm{D}-\mathrm{QSAR}$ is to determine the bioactive conformations of the compounds so that all compounds could be aligned together. In this study, the central thiazolylhydrazide moiety [OC-N-N$\mathrm{C}=\mathrm{N}-\mathrm{C}=\mathrm{C}-\mathrm{S}$; Fig. 1] commonly found in the PTH derivatives was used as the substructure for structural alignment. The most active compound $\mathbf{1}$ (Table 1) was used as a template for alignment, and 22 training set molecules and 5 test set molecules were all aligned together (Fig. 2).

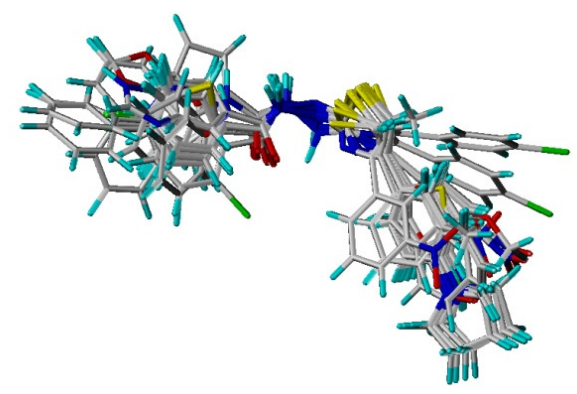

Figure 2. Structural alignment of the PTH derivatives around the common substructure $\mathrm{OC}-\mathrm{N}-\mathrm{N}-\mathrm{C}=\mathrm{N}-\mathrm{C}=\mathrm{C}-\mathrm{S}$. 
Table 1. PTH derivatives used for 3D-QSAR study

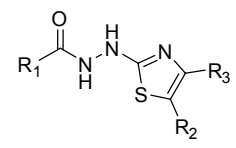

\begin{tabular}{lllll}
\hline Compd & $\mathrm{R}_{1}$ & $\mathrm{R}_{2}$ & $\mathrm{R}_{3}$ & $\mathrm{IC}_{50}(\mu \mathrm{M})$ \\
\hline
\end{tabular}

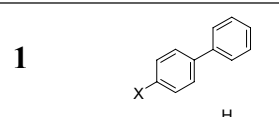

2

3

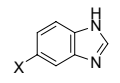

$\mathrm{H}$

$\mathrm{H}$

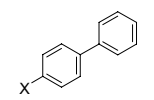

$4 *$

5

6

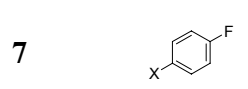

8*

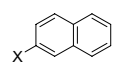

9

10

11

12

13

14

$15 *$

16

17

18

$19 *$

20

21

22

23

24

25

26

$27 *$

$\mathrm{H}$

H

$\mathrm{H}$

H

H

H

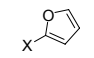

(1)

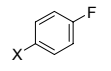

Cl

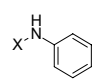

.

$x \in$

盛

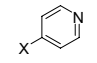

$x$

$x+\frac{\pi}{3}$

$\times \sqrt{18}$

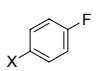

a

1
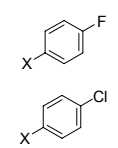

Sc1

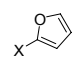

5

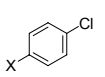

$\mathrm{H}$

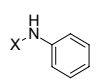

H

$\mathrm{H}$

H

$\mathrm{CH}_{3}$

$\mathrm{H}$

$\mathrm{H}$

H

H

H

H

H

H

H

H

H

H

$\mathrm{H}$

$\mathrm{NO}^{\mathrm{NO}_{2}}$
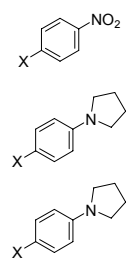

$\mathrm{Nin}^{\mathrm{NO}_{2}}$

$\mathrm{N}^{\mathrm{NO}_{2}}$
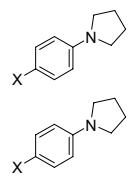

146.5

146.5

183.8
With the ligand alignment in hand, we attempted 3D-QSAR study by using CoMFA ${ }^{17,18}$ as well as CoMSIA ${ }^{19,20}$ method. Tripos standard CoMFA field was used for construction of a CoMFA model, whereas a CoMSIA model was built by using combinations of steric, electrostatic, hydrogen bond donor, hydrogen bond acceptor, and hydrophobic fields as descriptors. As biological activities are generally skewed, the reported $\mathrm{IC}_{50}$ values (concentration of a compound required to inhibit the tau aggregation by $50 \%$ ) were converted into the corresponding $\log \mathrm{IC}_{50}$. CoMFA and CoMSIA were set at standard values (with default grid spacing of $2.0 \AA$ ), with a $s p^{3}$ carbon atom with one positive charge used to probe steric and electrostatic fields. The standard cutoff value was set to $30 \mathrm{kcal} / \mathrm{mol}$. As usual, PLS (partial least squares) method was used to establish and validate 3D-QSAR, and LOO (leave-one-out) cross-validation method was used to evaluate the initial model. The cross-validated coefficient $q^{2}$ was calculated, the optimum number of components was then given, and the $3 \mathrm{D}$ model was finally derived corresponding to the optimum number. The column filtering box was kept unchecked during all operations, and the results obtained from the PLS analysis are summarized in Table 2.

The cross-validated correlation coefficient $\left(q^{2}\right)$ defines the goodness of prediction whereas the conventional (non-crossvalidated) correlation coefficient $\left(r^{2}\right)$ indicates goodness of fit of a QSAR model. SEP and SEE (Table 2) are statistical measures available for evaluation of the significance of the model and in both cases the smaller is the better. The $F$-test value (F, Table 2) also stands for statistical significance of the model (the higher, the better). Both the CoMFA model $\left(q^{2}=0.61, N=5\right)$ and CoMSIA method using steric as well as electrostatic field as descriptor variables $\left(\right.$ CoMSIA $\left._{\mathrm{se}}\right)\left(q^{2}=0.57, N=4\right.$, Table 2$)$ are determined to be statistically significant, but CoMFA field provided better model with $r^{2}$ of 0.98 (with $\mathrm{SEE}=0.10$ and $\mathrm{F}=$ 147.73; Table 2). The field distributions of the steric and electrostatic field descriptor variables were 48.4 and $51.6 \%$, respectively, which indicates that electrostatic and steric field contributes equally well to the final CoMFA model.

Graphical representations of CoMFA steric and electrostatic contour plots obtained by the field type "stDev*coeff" are displayed in Fig. 3. The contour maps were superimposed on the most active compound 1 . The green and blue regions in Fig. 3(a) and Fig. 3(b) indicate areas where steric bulk and electronegative groups enhance biological activity, respectively. On the other hand, the yellow and red contours indicate regions where steric bulk and electronegative groups are detrimental to biological activity. The PTH derivatives have tweezer-like structures with a pair of prongs (two aromatic rings) attached to a hinge (central thiazolylhydrazide functionality). The CoMFA contour plot (Fig. 3) shows that the binding sites of the receptor for these two aromatic rings are unsymmetrical. Among the two aromatic prongs, one attached to the hydrazide carbonyl carbon is allowed to adopt wide structural variations (dumbbell-shaped green contour in the left side of Fig. 3a) while the other attached to the thiazole ring is strictly confined in a small region of conformational space (green and yellow contours in the right side of Fig. 3a). In addition, substitution on the thiazolic aromatic ring is not favored, which is clearly seen by the red contours surrounding the right-hand side of the PTH derivative (Fig. 3b). 
Table 2. PLS analysis on PTH derivatives

\begin{tabular}{|c|c|c|c|c|c|c|c|c|}
\hline \multirow{2}{*}{ Model } & \multicolumn{3}{|c|}{ Cross-validated } & \multicolumn{3}{|c|}{ Non-cross-validated } & \multicolumn{2}{|c|}{ Fraction $\%$} \\
\hline & $q^{2}$ & SEP & $N$ & $r^{2}$ & SEE & $\mathrm{F}$ & $\mathrm{S}$ & $\mathrm{E}$ \\
\hline CoMFA & 0.61 & 0.44 & 5 & 0.98 & 0.10 & 147.73 & 48.4 & 51.6 \\
\hline CoMSIA $_{\mathrm{se}}{ }^{a}$ & 0.57 & 0.44 & 4 & 0.92 & 0.20 & 47.76 & 33.2 & 66.8 \\
\hline CoMSIA $_{\mathrm{da}}^{b}$ & 0.15 & 0.61 & 3 & $-d$ & $-_{-}^{d}$ & $-^{d}$ & $-_{-}^{d}$ & $-^{d}$ \\
\hline $\mathrm{CoMSIA}_{\mathrm{h}}{ }^{c}$ & 0.40 & 0.50 & 2 & $-d$ & $-d$ & $-d$ & $-d$ & $-d$ \\
\hline
\end{tabular}

$q^{2}$ : leave-one-out (LOO) cross-validated correlation coefficient, SEP: standard error of prediction, $N$ : optimum number of components, $r^{2}:$ non-crossvalidated correlation coefficient, SEE: standard error of estimate, F: F-test value, S: fraction of steric field, E: fraction of electrostatic field. ${ }^{a} \mathrm{~S}=$ steric field, $\mathrm{e}=$ electrostatic field. ${ }^{b} \mathrm{~d}=$ hydrogen bond donor field, $\mathrm{a}=$ hydrogen bond acceptor field. ${ }^{c} \mathrm{~h}=$ hydrophobic field. ${ }^{d}$ not determined.

(a)

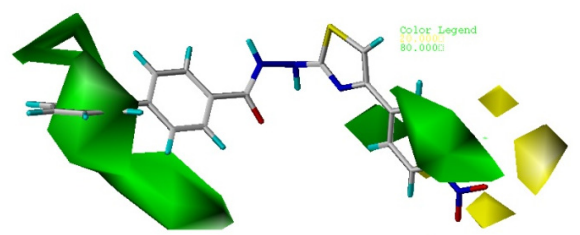

(b)

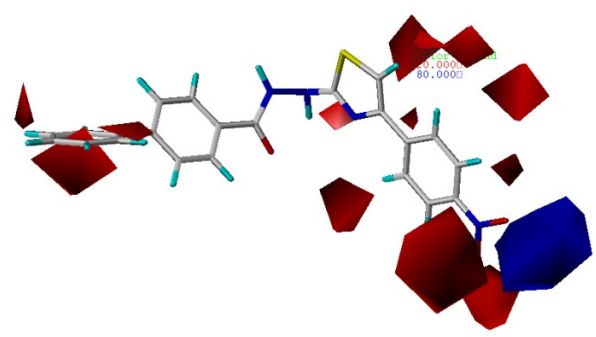

Figure 3. 3D contour maps around compound 1 as the result of CoMFA analysis of inhibition of tau aggregation by the PTH derivatives. (a) The color coding indicate regions where substitution enhances (green) or reduces (yellow) the anti-aggregative activity; (b) Regions where electronegative substituents enhance (blue) or reduce (red) the inhibition of tau aggregation.

(a)<smiles>O=C(OCCOC(=O)c1ccc([N+](=O)[O-])cc1)c1ccc(-c2ccccc2)cc1</smiles>

(b)

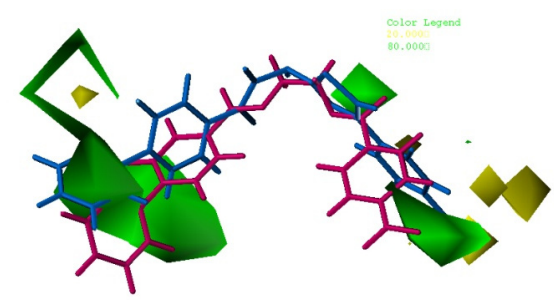

Figure 4. (a) Structure of the compound 28. (b) Steric contour map of the CoMFA model superimposed with the compounds 1 (in blue) and 28 (in red).

It is of particular interest that even the most active compound $\mathbf{1}$, superimposed with the contour plots in Fig.3, is not in perfect match with the sterically favored green contours located on the thiazole side of the central hydrazide moiety (green contours in right side of Fig. 3a), and the thiazolic aromatic ring is located between the two green contours.

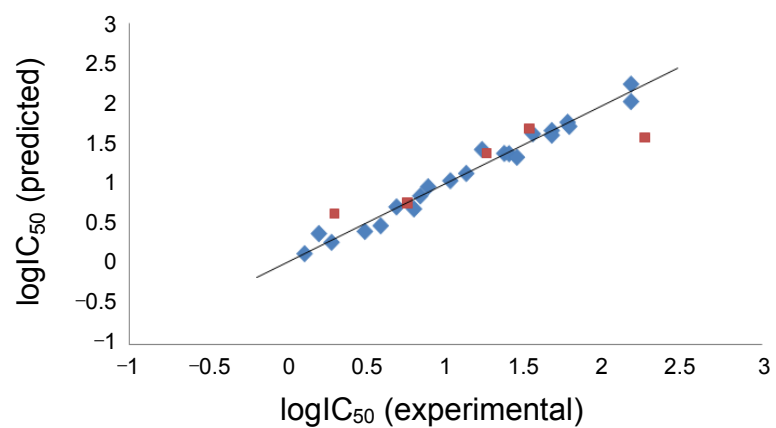

Figure 5. Plot of experimental versus CoMFA model-predicted $\log \mathrm{IC}_{50}$ of training set (diamond) and test set (rectangle).

Thus, it is fair to assume that the rigid thiazolylhydrazide hinge structure prevents PTH derivatives to adopt the most active conformation covering both sides of the sterically allowed regions at the same time. In this context, it is conceivable that more potent tau aggregation inhibitors can be designed by replacement of the central thiazolylhydrazide with a more flexible hinge. As anticipated, compound $\mathbf{2 8}$ with two aromatic rings (biphenyl and 4-nitrophenyl) connected with a flexible ethylene glycol linker (Fig. 4a) showed a perfect match with the steric contour map, in which the aromatic rings on both ends occupy the green contours (Compound in red, Fig. 4b). In particular, the aromatic ring of $\mathbf{2 8}$ corresponding to the thiazolic aromatic ring of $\mathbf{1}$ is located on the green contour. Also, as the thiazolylhydrazide moiety is a well known toxicophore, replacement of the thiazolylhydrazide with an ethylene glycol linker would help reduce the cytotoxicity of the PTH derivatives.

The predictive ability of the CoMFA models was assessed by predicting $\log \mathrm{IC}_{50}$ values of an external test set composed of 5 compounds $(\mathbf{4}, \mathbf{8}, \mathbf{1 5}, \mathbf{1 9}$, and 27; Table 2). The external validation process is considered the most reliable validation method, as the test set compounds are not included in the construction of the 3D-QSAR models. The results are listed in Table 3, and the graphic results for the experimental versus predicted activities of both training set and test set are displayed in Fig. 5. The good agreement between actual and predicted $\log \mathrm{IC}_{50}$ values for the test set compounds $\left[r^{2}\right.$ pred $=0.88$, residual $\left.(\delta)=-0.33 \sim 0.69\right]$ suggests that the constructed 3D QSAR models are reliable and can be used for the design of novel tau aggregation inhibitors. The CoMFA model also predicted significant tau aggregation inhibition by the proposed novel lead compound $\mathbf{2 8}\left(\operatorname{logIC} \mathrm{C}_{50}=\right.$ 0.22 , Table 3 ). 
Table 3. Comparison of experimental (Exp) and CoMFA model-predicted (Pred) inhibition of tau aggregation $\left(\operatorname{logIC} \mathrm{C}_{50}\right)$ by the PTH derivatives. Differences between the two values are represented by residuals $(\delta)$. Data shaded in this table belong to the test set compounds $(\mathbf{4}, \mathbf{8}$, 15, 19, and 27).

\begin{tabular}{|c|c|c|c|}
\hline \multirow{2}{*}{ Compd } & \multicolumn{2}{|c|}{$\log \mathrm{IC}_{50}$} & \multirow{2}{*}{$\delta$} \\
\hline & Exp & Pred & \\
\hline 1 & 0.11 & 0.13 & -0.02 \\
\hline 2 & 0.20 & 0.38 & -0.18 \\
\hline 3 & 0.28 & 0.27 & 0.01 \\
\hline 4 & 0.30 & 0.63 & -0.33 \\
\hline 5 & 0.49 & 0.41 & 0.08 \\
\hline 6 & 0.59 & 0.48 & 0.11 \\
\hline 7 & 0.69 & 0.72 & -0.03 \\
\hline 8 & 0.76 & 0.76 & 0.00 \\
\hline 9 & 0.80 & 0.69 & 0.11 \\
\hline 10 & 0.84 & 0.85 & -0.01 \\
\hline 11 & 0.89 & 0.97 & -0.08 \\
\hline 12 & 1.03 & 1.04 & -0.01 \\
\hline 13 & 1.13 & 1.13 & 0.00 \\
\hline 14 & 1.23 & 1.43 & -0.20 \\
\hline 15 & 1.26 & 1.39 & -0.13 \\
\hline 16 & 1.37 & 1.38 & -0.01 \\
\hline 17 & 1.40 & 1.38 & 0.02 \\
\hline 18 & 1.45 & 1.34 & 0.11 \\
\hline 19 & 1.53 & 1.70 & -0.17 \\
\hline 20 & 1.55 & 1.63 & -0.08 \\
\hline 21 & 1.67 & 1.67 & 0.00 \\
\hline 22 & 1.67 & 1.61 & 0.06 \\
\hline 23 & 1.77 & 1.77 & 0.00 \\
\hline 24 & 1.78 & 1.72 & 0.06 \\
\hline 25 & 2.17 & 2.03 & 0.14 \\
\hline 26 & 2.17 & 2.25 & -0.08 \\
\hline 27 & 2.26 & 1.57 & 0.69 \\
\hline 28 & - & 0.22 & - \\
\hline
\end{tabular}

In summary, a 3D-QSAR study on the PTH derivatives was performed in anticipation of getting a model that would account for the quantitative differences in inhibitory activity against the tau protein aggregation seen in this series and provide insights into designing of novel tau aggregation inhibitors with improved activity and reduced cytotoxicity. A 3D-QSAR model was constructed by using 22 PTH derivatives and, on the basis of a common thiazolylhydrazide structural motif, a stable and predictive CoMFA model with acceptable $q^{2}$-value was developed. The CoMFA 3D-QSAR model along with the information gathered from the $3 \mathrm{D}$ contour maps indicated that the relative orientation of the two aromatic rings $\left(\mathrm{Ar}_{1}\right.$ and $\mathrm{Ar}_{2}$, Fig. 1) attached to both ends of the tweezer-like structure of the PTH is the key to its biological activity. Also, by replacement of the rigid and toxicophoric thiazolylhydrazide moiety with a more flexible ethylene glycol linker, we showed that the two aromatic rings attached to the central ethylene glycol functionality can adopt the most favorable conformation for inhibition of tau protein aggregation, which warrants extensive structure-activity relationship study around the thiazolylhydrazide moiety to develop more potent and safe tau aggregation inhibitors.

Acknowledgments. This work was supported by a grant from Agenda Program (NIAS, 8-20-52), a grant from Biogreen 21 (Korea Ministry of Agriculture and Forestry), and by Priority Research Centers Program through the National Research Foundation of Korea (NRF) funded by the Ministry of Education, Science and Technology (2009-0093824).

\section{References}

1. Hardy, J.; Selkoe, D. J. Science 2002, 297, 353.

2. Vieira, M. N.; Forny-Germano, L.; Saraiva, L. M.; Sebollela, A.; Martinez, A. M.; Houzel, J. C.; De Felice, F. G.; Ferreira, S. T. J. Neurochem. 2007, 103, 736.

3. Leroy, K.; Bretteville, A.; Schindowski, K.; Gilissen, E.; Authelet, M.; De Decker, R.; Yilmaz, Z.; Buee, L.; Brion, J. P. Am. J. Pathol. 2007, 171, 976.

4. Ballatore, C.; Lee, V. M.; Trojanowski, J. Q. Nat. Rev. Neurosci. 2007, 8, 663 .

5. Xia, W. Curr. Opin. Investig. Drugs. 2003, 4, 55.

6. Wischik, C. M.; Bentham, P.; Wischik, D. J.; Seng, K. M. Alzheimer's and Dementia 2008, 4, T167.

7. Bulic, B.; Pickhardt, M.; Khlistunova, I.; Biernat, J.; Mandelkow, E. M.; Mandelkow, E.; Waldmann, H. Angew. Chem. Int. Ed. 2007, 46, 9215

8. Pickhardt, M.; von Bergen, M.; Gazova, Z.; Hascher, A.; Biernat, J.; Mandelkow, E.-M.; Mandelkow, E. Curr. Alzheimer Res. 2005, 2, 219.

9. Larbig, G.; Pickhardt, M.; Lloyd, D. G.; Schmidt, B.; Mandelkow, E. Curr. Alzheimer Res. 2007, 4, 315.

10. Pickhardt, M.; Larbig, G.; Khlistunova, I.; Coksezen, A.; Meyer, B.; Mandelkow, E.-M.; Schmidt, N.; Mandelkow, E. Biochemistry 2007, 46, 10016.

11. Pickhardt, M.; Biernat, J.; Khlistunova, I.; Wang, Y. P.; Gazova, Z.; Mandelkow, E. M.; Mandelkow, E. Curr. Alzhemier Res. 2007, 4, 397.

12. Pickhardt, M.; Gazova, Z.; von Bergen, M.; Khlistunova, I.; Wang, Y.; Hascher, A.; Mandelkow, E. M.; Biernat, J.; Mandelkow, E. J. Biol. Chem. 2005, 280, 3628.

13. Necula, M.; Chirita, C. M.; Kuret, J. Biochemistry 2005, 44, 10227.

14. Taniguchi, S.; Suzuki, N.; Masuda, M.; Hisanaga, S.; Iwatsubo, T.; Goedert, M.; Hasegawa, M. J. Biol. Chem. 2005, 280, 7614.

15. Kazius, J.; McGuire, R.; Bursi, R. J. Med. Chem. 2005, 48, 312.

16. SYBYL 7.2 Tripos International, 1699 South Hanley Rd., St. Louis, Missouri, 63144, USA.

17. Cramer, M.; Cramer, R. D.; Jones, D. M. J. Am. Chem. Soc. 1988, $110,5959$.

18. Kim, J.; Lee, M.; Kang, S. -Y.; Park, J.; Lim, Y.; Koh, D.; Park, K. H.; Chong, Y. Bull. Korean Chem. Soc. 2006, 27, 1025.

19. Klebe, G.; Abraham, U.; Mietzner, T. J. Med. Chem. 1994, 37, 4130.

20. Kim, J.; Han, J. H.; Chong, Y. Bull. Korean Chem. Soc. 2006, 27 , 1919. 